\title{
LA GRAMMAIRE DU FRANÇAIS ET SON HISTORIOGRAPHIE: APPRÉHENSIONS, À DEUX SIÈCLES DE DISTANCE, D'UN OBJET DE DÉBATS
}

Dans cet article, consacré à l'historiographie de la grammaire française, on abordera d'abord le problème de la définition du champ de l'histoire (ou de l'historiographie) de la grammaire française (et de la linguistique française).

La deuxième partie du texte sera consacrée à un problème central de l'épistémologie historique de la grammaire française: celui de la "récurrence du fait historique", problème que je voudrais analyser à travers un cas concret, celui de l'accueil fait à la grammaire de l'Académie.

\section{L'historiographie de la grammaire/de la linguistique: la question de l'objet}

On a tenté de concevoir et, parfois, de définir (ou d'expliciter) l'historiographie de la grammaire/linguistique comme étant dans un rapport d'iconicité partielle avec un objet appelé "l'histoire de la linguistique". Une telle attitude permet d'éluder la difficulté de devoir définir l'objet ou le domaine de cette discipline. Que cet objet ne soit guère donné d'avance, cela ressort déjà de l'histoire même de l'historiographie de la linguistique: il suffira de comparer à ce propos des travaux comme ceux de Thomsen (1902) et de Pedersen $(1916,1924)$ à ceux de Robins (1979²) ou de Mounin (1967). En fait, cela ne doit guère nous étonner: comme l'historiographie de la linguistique est une représentation de connaissances à propos de l'histoire, il est inéluctable que la notion de son objet varie, du moins dans son extension, dans la mesure où les connaissances à propos de l'histoire de la linguistique se sont élargies, enrichies ou modifiées.

La délimitation difficile de l'historiographie de la linguistique tient au fait que comme toute discipline histor[iograph]ique, elle est une science "totalitaire", au sens défini par Greimas: science dont le contenu est "la totalité des significations humaines" (Greimas 1976: 162), contenu restreint ici par l'adjonction du complément déterminatif de la grammaire/linguistique ${ }^{1}$. L'absence de procédure de délimitation, résultant d'un indéfini "extensionnel" (où s'arrête l'histoire ?) et d'un indéfini "intensionnel" (comment définir ce qui est/sera l'objet d'une histoire de la grammaire/linguistique?),

* Nos citations de textes respectent la graphie et la ponctuation de l'original. Pour les abréviations utilisées dans la deuxième partie du texte, voir note 24 .

1 Cf. Brekle (1985: 6-7): "Sollen nun aber tatsächlich möglichst alle Fragen, die jemals an die Sprache oder an einzelne Sprachen gestellt worden sind, in den Gesamtbereich der Sprachwissenschaftsgeschichte aufgenommen werden, so heisst dies, dass der Sprachwissenschaftshistoriker alle Zeugnisse, alle in Bezug auf Sprachreflexion 
n'est pas une raison suffisante pour justifier le silence qui s'est fait sur le problème. On ne peut que regretter l'attitude naïve caractéristique de la plupart des histoires de la linguistique: c'est comme si l'objet de l'histoire de la linguistique se présentait avec une évidence écrasante. Le caractère problématique d'une telle attitude apparaît aussitôt dès qu'on s'interroge sur certaines inclusions ou exclusions d'objets (possibles). En effet, une telle interrogation - invitation indirecte à s'ouvrir à ce qu'on ne connaît pas ou à ce qu'on ne veut pas connaître - force l'historiographe de la linguistique à expliciter son attitude à l'égard de la prise en compte éventuelle d'objets qui peuvent relever ou qui relèvent (aussi) d'autres disciplines, comme par ex.

(a) les réflexions philosophiques sur la nature et la fonction du langage, sur "langue(s), homme, société et Weltbild', sur langue, science et philosophie, sur le langage comme technique philosophique.

(b) les apports, à travers l'histoire, de nature "sémantique", qui relèvent (en premier lieu) de la logique, comme par ex. la théorie de la suppositio, l'analyse des termes d'un jugement, la constitution de la notion de "référence" (ou "dénotation"), la construction (ou la désarticulation) logique de systèmes symboliques. Comment justifier la place qu'on donnera (ou qu'on ne donnera pas) à Petrus Hispanus, à Vincent Ferrer, à Boole, à Frege, à Russell et Whitehead, ou à Peirce dans une histoire de la linguistique?

(c) l'emploi de l'écriture - en tant que système de communication sémiotique à côté du langage oral - à travers l'histoire. La naissance et l'évolution des systèmes d'écriture ont fait l'objet d'études particulières ${ }^{2}$, et celles-ci ont dégagé le progrès structurel qui caractérise le passage des systèmes d'écriture "idéographique" aux systèmes alphabétiques. Il est significatif que ce passage s'effectue presque simultanément à différents endroits dans le Proche-Orient et dans le Moyen-Orient, sans qu'on puisse repérer des influences directes déterminantes. Gelb a invoqué comme explication une "diffusion de stimuli", et cela correspond à un raffinement de l'analyse des unités du système linguistique, qu'on parvient à transcoder dans des séries de formes graphiques plus facilement "maîtrisables". La réduction de la production orale à un système de notation est non seulement une remarquable invention ou une vraie "révolution" dans l'histoire des cultures: elle est aussi le fondement de toute analyse linguistique. Stade initial de l'histoire de la linguistique, les systèmes d'écriture ont pourtant été largement négligés par les historiens de la linguistique ${ }^{3}$.

deutbaren Quellen als in seinen Forschungshorizont fallend wird berücksichtigen müssen", et ibid., p. 19: "Statt dessen erscheint es doch wohl angemessener, die uns überlieferten Texte in einem systematischen Interpretationsund Rekonstruktionsprozess so zu behandeln, dass am Ende der heutige Sprachwissenschaftler in der Lage ist, zu erkennen, aufgrund welcher Prämissen, Implikationen etc., mit welchen Interessen, mit welcher Methode ein früherer Sprachwissenschaftler, Grammatiker oder ganz einfacher Mensch sich Gedanken über sprachliche Phänomene gemacht hat".

2 Voir par exemple: Février (1948), Cohen (1958), Diringer (1948, 1962) et Gelb (1969).

3 On n'oubliera pas non plus que l'écriture véhicule un certain nombre de fonctions symboliques (associées avec certains types de textes ou de supports matériels: amulettes magiques à inscription, tablettes d'exécra- 
(d) les systèmes cryptographiques, permettant de transcoder des messages (oraux/écrits), en limitant de façon consciente l'interprétation de ces messages à des récepteurs connaissant le système et les principes d'encodage.

Le problème de la délimitation reçoit un début de solution si l'on se dégage d'une vue trop extensionnaliste (qui impliquerait que pour tel objet $x$, on saurait dire s'il appartient oui ou non au domaine de recherche) et si on se place au niveau de l'histoire comme reconstruction de contenus signifiants. Dans une telle optique, l'historiographie de la grammaire/linguistique a pour but de décrire comment le savoir linguistique s'est développé: quelles sont les formes prises par la connaissance linguistique (on n'écrit plus une grammaire d'une langue comme le faisait par ex. Pānini), comment les connaissances linguistiques ont-elles pris naissance 4 (comment faut-il "expliquer" que c'est seulement au $18^{\mathrm{e}}$ siècle que Bonamy 5 a reconnu dans le latin vulgaire l'ancêtre du français et des autres langues romanes?), comment se sont-elles développées (comment a-t-on précisé la nature et l'action des laryngales en indo-européen?), comment ces connaissances ont-elles été communiquées et à quel public (la "communication linguistique" est aux $16^{\mathrm{e}}-18^{\mathrm{e}}$ siècles une affaire de philosophes, d'historiens, d'érudits et de grammairiens; à partir du $19^{\mathrm{e}}$ siècle, on assiste à une "professionnalisation" de la discipline).

L'historiographie de la grammaire/linguistique a donc un référent historique. On évitera toutefois de l'identifier comme un "fait" discret (un énoncé $E$ produit par un linguiste à un moment $t_{o}$ ), repérable en dehors de toute contextualisation. On évitera davantage de l'identifier avec un nom, un titre, ou une date (de publication). Il s'agit là de vues appauvrissantes qui passent à côté de l'essence de la science: celle-ci est un processus de modélisation cognitive, par laquelle des "agents" ou "actants" abordent un certain nombre de "problèmes" dans un "contexte" donné. Cette structure événementielle est récurrente et elle peut être considérée comme un invariant structurel, si on admet l'idée qu'il existe une sorte de "structure profonde" de l'histoire. Cette structure comporte un grand nombre de variables, que nous essaierons de détailler ci-après.

\section{(I) variables affectant les "actants"}

Placés dans le temps, les actants possèdent des connaissances linguistiques, historiques et autres qui ont une détermination temporelle. Il serait faux d'en chercher l'ex-

tion, marques d'esclaves, etc.), qui présentent un intérêt pour l'historien de la linguistique. De même, la maîtrise de l'écriture était fortement valorisée dans les cultures anciennes (cf. par exemple le culte du métier de scribe dans l'Égypte ancienne).

4 Ce type de questions préoccupait avant tout les premiers historiens de la linguistique. Cf. F. Thurot, Tableau des progrès de la science grammaticale (1796), p. 66: "L'histoire de l'origine de la science grammaticale présente le plus grand intérêt; et s'il était possible d'y porter un degré suffisant d'exactitude, et de lui donner un caractère d'authenticité, qui pût satisfaire les bons esprits, cette histoire serait le meilleur livre élémentaire que l'on pût avoir sur la grammaire, et en même temps un excellent traité de philosophie, puisqu'elle seroit aussi l'histoire de nos idées".

5 Voir les textes de P.-N. Bonamy réunis dans Albrecht (éd. 1975). 
plication dans une "rupture épistémologique": on expliquerait une situation par son résultat. La "rupture" même s'explique par des chaînes de situations, de faits et d'événements.

À côté de variables purement individuelles (intelligence, intérêts, tempérament, etc.), des variables plus générales - rapport avec des (types de) langues, avec une certaine pratique de l'enseignement (à quel niveau?, sous quelles formes?) - affectent le comportement des agents scientifiques.

\section{(II) variables affectant les "problèmes"}

Il est significatif qu'à travers l'histoire de la grammaire/linguistique des problèmes variables ont été au centre des préoccupations des savants: l'adéquation ou la nonadéquation du langage (par rapport à la réalité, ou par rapport à nos représentations mentales), la maîtrise des structures grammaticales de la langue maternelle, la "généalogie" des langues, la nature de la compétence grammaticale, etc. La variabilité des problèmes est d'une part liée à des variables affectant les actants (comme par ex. $1^{\mathrm{e}}$ rapport avec un certain type de langues ou les intérêts spécifiques) et d'autre part à des variables qui relèvent du contexte (l'évolution des sciences en général; le degré d'institutionnalisation de la linguistique, etc.), mais la prépondérance massive d'un type de problèmes à une époque déterminée (par ex. la production de grammaires descriptives à l'époque de la Renaissance; l'importance de la grammaire générale au $18^{e}$ siècle; le rôle dominant de la grammaire historico-comparative au $19^{e}$ siècle) ne s'explique pas uniquement par le contexte.

Il semble qu'il existe aussi une dynamique interne de ces problèmes et de leur importance respective: cette dynamique a bien sûr des assises contextuelles, mais le facteur principal, et très difficilement contrôlable, est la valorisation des problèmes: cette valorisation est plus massive dans les périodes où (a) la linguistique compte un nombre plus réduit d'agents, et (b) possède un circuit de communication plus clos (et plus homogène). Il est par exemple significatif que la grammaire médiévale ne pose pas, avant le $12^{\mathrm{e}}$ siècle, le problème de la correspondance entre catégories grammaticales et catégories mentales et "réelles". Expliquer l'émergence de la grammaire spé-culative (dont l'apparition est massive à Paris au $13^{\mathrm{e}}$ siècle) par l'influence de l'aristotélisme ou par la contagion d'une abondante production en logique sémantique (ou philosophie de la logique: littérature des sophismata, traités sur la suppositio des termes) n'est point suffisant: la pensée d'Aristote était déjà connue, partiellement il est vrai, au haut Moyen Âge et le détail même de l'exposé des modistes n'est pas calqué sur l'enseignement d'Aristote. D'autre part, c'est la "co-présence" de la grammaire spéculative et d'une sémantique logique qu'il faut expliquer.

La dynamique interne des problèmes est une donnée difficile à saisir, d'une part parce qu'elle ne se manifeste pas partout avec la même intensité ni au même moment, d'autre part parce que cette dynamique est celle d'un objet à plusieurs faces, qui se prête à différents "abordages", de sorte qu'il est rare qu'un problème s'impose avec exclu- 
sivité. La difficulté de saisir la dynamique réside avant tout dans le fait qu'il s'agit d'un phénomène (a) processuel (b) à base cognitive. La première caractéristique implique la comparaison d'états de la science, ou le dépassement constant d'un corpus établi de textes; la seconde caractéristique exige qu'on s'intéresse au savoir linguistique non pas seulement du point de vue de ses résultats (c'est d'ailleurs un point de vue trompeur: un résultat, c'est une réponse qu'on identifie par rapport à une question qu'on pose, par ex., à un texte linguistique du $17^{\mathrm{e}}$ siècle; un tel "résultat" est en fait un résidu par rapport à ce que le texte est dans son repli sur le $17^{\mathrm{e}}$ siècle) ${ }^{6}$, mais plutôt du point de vue de leur envisagement: quels sont les problèmes qu'on a envisagés et comment les a-ton envisagés?

Ces questions conduisent, presque inéluctablement, à une vue de la dynamique des sciences comme procédant par des "métaphorisations"7, ou plutôt des transferts coneptuels (souvent à base imaginative très forte). Une telle vue de l'évolution des sciences (comme on la trouve chez Toulmin) ${ }^{8}$ présente deux avantages:

(a) elle rend justice au fait que la science repose toujours sur (un emploi particulier) du langage (cf. les travaux de Granger) ${ }^{9}$;

(b) elle explique la continuité naturelle entre mythologie, idéologie et science.

Ce transfert conceptuel est en fin de compte un processus de perception d'analogies, de comparaison à travers des ensembles non identiques entre lesquels on établit une certaine homogénéité:

"All formation of new concepts, all change in concepts, involves discovery of the world that is, the development of a new way of looking at the world (reflected in statements about the way the world is), which may be more or less borne out as time goes on. Every theory of the formation of new concepts is also about discovering the way the world is (...) Metaphors, in this sense, are the traces left by the displacement of concepts. They bear witness to complex processes of displacement of concepts over time just as present living species bear witness to biological evolution (...) But the displacement begins with the intimation of such a similarity and may be justified after the fact by pointing out the similarity in terms which are themselves results of displacement. Observation of analogies is the result and partial justification of the displacement of concepts" (Schon 1963: 36 et 41).

6 En d'autres termes, ce qu'un texte ne dit pas par rapport à la question (ou aux questions) qu'on lui pose (aujourd"hui), est souvent un "résultat" dans sa fonction authentique.

7 Dans Swiggers (1991), nous avons distingué trois types de métaphorisation: la métaphorisation plate (celleci permet de créer un terme/concept, par simple association avec un domaine qui ne possède pas la même structure que le domaine étudié et qui ne peut guère fonctionner comme modèle conceptuel global de ce dernier), la métaphorisation active (qui crée des termes opérationnels tout en activant le domaine adjacent où on a pris le terme, comme un modèle possible du domaine auquel on l'applique), et la métaphorisation interne (correspondant aux cas de conceptualisation qui ont leur origine dans une schématisation des structures grammaticales, ou dans une visée de la langue).

8

Cf. Toulmin (1960).

9 Cf. Granger $(1960,1968)$. 
- (a) Le transfert conceptuel peut s'appliquer à l'objet "langue" dans son intégralité, ou bien à une manifestation particulière de cet objet. Si l'on prend le premier cas, on peut identifier, au cours de l'histoire de la grammaire/linguistique, au moins les "envisagements" suivants de l'objet "langue":

(a) la langue comme moyen d'expression d'un contenu mental: ici, la langue est appréhendée comme un instrument de signification, dont le point de départ est une représentation conceptuelle;

(b) la langue comme ensemble de formes linguistiques: cet ensemble peut être envisagé dans un sens très lâche, comme agglomérat d'unités hétérogènes (cf. la grammaire de Denys le Thrace);

(c) la langue comme objet historique, rattachable à un antécédent perdu (cf. la généalogie des langues);

(d) la langue comme un ensemble de formes qui articulent l'analyse de la pensée: ce type d'approche est une intégration des approches (a) et (b);

(e) la langue comme ensemble de formes, constituant des correspondances "latérales" et "verticales", qu'on peut situer dans le temps: c'est l'approche de la grammaire historico-comparative aux $19^{\mathrm{e}}$ et $20^{\mathrm{e}}$ siècles;

(f) la langue comme capacité organique permettant d'articuler une vision du monde (cf. Humboldt);

(g) la langue comme convention sociale, n'existant que sous la forme d'une abstraction par rapport à ses exploitations discrètes, partielles et dispersées dans la communauté (cf. F. de Saussure);

(h) la langue comme structure autorégulatrice et hiérarchisée, dont l'analyse par niveaux correspond à des techniques précises de description;

(i) la langue comme modélisation symbolique: la langue est un ensemble de signes par lequel le sujet humain domine la réalité et élabore son rôle de "participant" (à la réalité, à la vie en communauté);

(j) la langue comme objet mathématisable (par ex. comme chaîne de symboles): ici, la langue est un type construit, auquel on peut "appliquer" des analyses probabilistes qui concernent par ex. son statut comme structure d'information;

(k) la langue comme un jeu ${ }^{10}$, dont les pièces constitutives sont les signes linguistiques, qui permettent plusieurs types d'emploi (cf. la conception de Wittgenstein);

(1) la langue comme compétence intériorisée, connaissance privée qui concerne l'exploitation - licite ou abusive - de règles grammaticales (règles d'ajout, de sélection, de déplacement, de suppression, etc.).

Cette liste n'est sans doute pas exhaustive, et elle est trop peu précise à plusieurs égards:

10 Ou plus généralement, comme moyen d'action (ce qui permet d'insérer la théorie du langage dans une théorie de l'action humaine). 
(i) Nous n'avons pas rendu justice à la variété des termes qui désignent l'objet langue dans ces différentes conceptions;

(ii) Il conviendrait de distinguer, là où il y a lieu, entre la langue comme objet de description et la langue comme notion non opérationnelle: certains envisagements - comme par ex. ceux présentés par Harris - distinguent explicitement l'objet théorique construit (par le grammairien) ${ }^{11}$, qui résulte d'une caractérisation de l'objet décrit, et cet objet "observable" (non idéalisé);

(iii) Il faudrait marquer les multiples combinaisons qui existent entre ces différentes conceptions;

(iv) Il faudrait préciser et raffiner la liste en ajoutant, là où il y a lieu, des modèles théoriques qui se différencient à l'intérieur d'un même envisagement.

- (b) À côté du transfert conceptuel opérant à une échelle globale, il existe aussi des transferts conceptuels affectant des "manifestations particulières": manifestations sous forme de structures, de propriétés ${ }^{12}$, etc. Les structures et les propriétés peuvent être "envisagées" selon des modelages conceptuels variables: il suffit de penser à certaines métaphorisations appliquées à des structures typologisées (cf. l'approche de structures expressives de la possession ou de la détermination/spécification chez $\mathrm{Seiler}^{13}$ en termes de "réponse à un problème (mental)"; ou l'approche de structures actancielles et syntactiques (positionnelles) chez Thom ${ }^{14}$ en termes de schémas d'appréhension et de flux communicatif), ou aux métaphorisations appliquées à certaines propriétés (certaines d'entre elles - comme par ex. analogue vs transpositif, renvoyant à la correspondance ou non-correspondance avec l'ordre des idées, ou primitif vs développé résultent déjà de transferts conceptuels: ainsi l'opposition entre le caractère synthétique des langues indo-européennes classiques et la nature analytique de leurs descendants a souvent été expliquée par un processus de dégénération).

\section{(III) variables affectant le "contexte"}

Toute vue sur le langage - qu'elle s'articule sous forme d'une théorie ou non s'insère dans un contexte. Nous avons déjà abordé un aspect de ce contexte, à savoir

11 Cf. Harris (1965: 9): "A grammar of a language seeks to show how all the sentences which would be accepted (under one or another criterion of acceptance [...]) can be characterized as particular types of combinations of particular classes of elements (phonemes, morphemes, words, sequences of words, sentences)". Voir les définitions que Harris y donne de la "String Analysis", de la "Immediate Constituents Analysis" et de la "Transformational Analysis".

Une telle vue s'accommode très bien de la distinction épistémologique faite par M. Bunge (1974) entre les deux référents d'une théorie: la théorie renvoie d'une part à un référent interne [direct] (l'objet construit par le modèle théorique), et d'autre part à un réfërent externe [indirect] (la classe des objets correspondant à l'extension de l'objet construit, en tant que type intensionnel).

12 L'opposition entre structures et propriétés n'est pas absolue: dans le cas où on superpose une paramétrisation à des structures observées, on peut décrire celles-ci comme des propriétés d'un type (ou sous-type) construit.

13 Cf. Seiler (1977, 1988).

14 Cf. Thom (1974). 
celui par lequel les actants se rattachent à un contexte déterminant certaines formes de connaissances. Dans cette section, nous voudrions évoquer d'autres aspects, qui relèvent du contexte institutionnel et culturel.

Parmi ces variables, il convient de mentionner en premier lieu le type de société et les pratiques d'enseignement qu'elle véhicule: on peut penser ici aux liens entre par ex. la grammaire sanskrite et la société ritualisée de l'Inde ancienne; entre l'activité des grammairiens hébreux et arabes et le souci de préserver, dans une forme orale intacte, le texte des livres sacrés; entre la production de grammaires latines élémentaires dans la basse Antiquité et le haut Moyen Âge et l'imposition du latin dans des régions habitées par des peuples ne parlant pas le latin et placés devant l'obligation d'apprendre le latin pour des raisons politiques, administratives et économiques; entre la forme et le contenu des grammaires spéculatives et le type d'enseignement, à base dialogale, prodigué dans les universités européennes, etc.

À côté de cette détermination sociale "extérieure", il faut mentionner le rôle du contexte intellectuel, le climate of opinion. Un aspect essentiel de ce "climat d'opinion" est ce que Michel Foucault ${ }^{15}$ a défini comme l'épistémé d'une époque. Pour Foucault, il s'agit d'une façon (peu explicite) d'ordonner les choses. Ces codes interprétatifs de la culture peuvent changer d'une époque à l'autre. Ainsi, au $17^{\mathrm{e}}$ siècle, la culture appréhende la réalité essentiellement par une théorie (statique) de la représentation; ce n'est qu'à partir de la fin du $18^{\mathrm{e}}$ siècle ou du début du $19^{\mathrm{e}}$ siècle que la culture occidentale constitue l'historicité des objets réels, en leur prêtant une vie organique ${ }^{16}$. Pour Foucault, cette recherche "archéologique" qui prend comme objet les théories de l'ordonnance des choses, et l'évolution des codes du langage, de la perception et de la pratique - ces codes sont la région médiane entre l'ordre empirique et les sciences - est essentiellement un exercice de comparaison épistémologique.

Ce savoir général n'est pas facilement repérable, vu qu'il est dispersé: on le retrouve dans les courants philosophiques, dans les attitudes générales des savants, dans les thèmes littéraires, etc. Mais son action est pénétrante et efficace. Ainsi, par exemple, le $18^{\mathrm{e}}$ siècle est un siècle où les thèmes littéraires sont des questions ou principes

15 Voir Foucault $(1966,1969)$.

16 Cf. Foucault (1966: 13-14): "Nous avons beau avoir l'impression d'un mouvement presque ininterrompu de la ratio européenne depuis la Renaissance jusqu'à nos jours, nous avons beau penser que la classification de Linné, plus ou moins aménagée, peut en gros continuer à avoir une sorte de validité, que la théorie de la valeur chez Condillac se retrouve pour une part dans le marginalisme du XIX ${ }^{e}$ siècle, que Keynes a bien senti l'affinité de ses propres analyses avec celles de Cantillon, que le propos de la Grammaire générale (tel qu'on le trouve chez les auteurs de Port-Royal ou chez B[e]auzée) n'est pas si éloigné de notre actuelle linguistique - toute cette quasi-continuité au niveau des idées et des thèmes n'est sans doute qu'un effet de surface; au niveau archéologique, on voit que le système des positivités a changé d'une façon massive au tournant du XVIII ${ }^{\mathrm{e}}$ et du XIX $\mathrm{X}^{\mathrm{e}}$ siècle. Non pas que la raison ait fait des progrès; mais c'est que le mode d'être des choses et de l'ordre qui en les répartissant les offre au savoir a été profondément altéré. Si l'histoire naturelle de Tournefort, de Linné et de Buffon a rapport à autre chose qu'à elle-même, ce n'est pas à la biologie, à l'anatomie comparée de Cuvier ou à l'évolutionnisme de Darwin, c'est à la grammaire générale de B[e]auzée, c'est à l'analyse de la monnaie et de la richesse telle qu'on la trouve chez Law, chez Véron de Fortbonnais ou chez Turgot". 
éthiques (par ex. l'éducation; le statut de la loi naturelle; le droit social; la corruption des mœurs), des problèmes philosophiques (par ex. le rapport entre langue et pensée; l'expression symbolique) ou historico-culturels (par ex. l'évolution des sociétés). En matière de sciences, c'est un siècle qui voit s'introduire des sciences théoriques fondées sur la notion de manipulation systématique de symboles: c'est le cas du calcul et de la théorie des fonctions. Les sciences empiriques sont formalisées ou même axiomatisées: c'est le cas de la mécanique rationnelle (d'Alembert, Traité de dynamique, 1743) et de la mécanique analytique (Lagrange, Mécanique analytique, 1788). De nouvelles disciplines voient le jour: elles concernent la conduction de l'électricité (et la conductivité relative des substances), le magnétisme, et les corps gazeux. Des progrès conceptuels importants sont réalisés en biologie et en physiologie animale. Buffon, procédant de façon empirique, rejette la classification linnéenne par genre et espèce, et défend un transformisme modeste (attribuant la modification des espèces au climat, à la nourriture et à la domestication), que Maupertuis radicalise vers un transformisme intégral (Essai sur la formation des corps organisés, 1754). La préexistence des genres (= préformisme) est abandonnée comme axiome après les découvertes de Trembley, Needham et C. F. Wolff. En physiologie animale, les savants découvrent des structures importantes: la digestion, la contraction musculaire, le système nerveux.

L'intérêt pour les principes, le goût de la structuration axiomatique, le souci de contrôler les généralisations par une extension des matériaux empiriques, sont des caractéristiques qu'on retrouve en matière de grammaire et de linguistique: les grammairiens du $18^{\mathrm{e}}$ siècle essaient de formuler des principes (cf. le titre de la grammaire de Girard: Les Vrais Principes de la langue françoise: ou la parole réduite en méthode, conformément aux loix de l'usage, 1747) et ces principes servent souvent à saisir un phénomène mouvant (ainsi Buffier [1709] propose-t-il le terme de "modification" pour décrire des phénomènes de nature très diverse: extension syntagmatique, par ex. par l'ajout d'un adverbe incident au prédicat verbal; intégration de séquences au noyau nominal et verbal, par ex. par l'ajout d'un complément déterminatif ou d'une phrase complétive) ${ }^{17}$; les grammairiens s'appliquent à axiomatiser (une partie) des domaines grammaticaux, et cet effort se manifeste d'abord au niveau de la mise en place d'un métalangage clos, stipulativement défini: c'est ce que Girard essaie de faire pour les concepts parole, pensée, idées, mot, valeur, ou langue, usage, dialecte, patois (Girard 1747: 18-22):

"Mais si les parures ne sont ici que d'un médiocre avantage; il n'en est pas demême de la netteté, de l'ordre, \& de l'exactitude: le sujet les exige dans le dernier degré; il faut que l'exposition en soit claire, la conduite commode, \& le détail expliqué dans toute son étendue quoiqu'avec précision. Je ne saurois donc mieux entrer en matiere que par des définitions faites avec soin, en commençant dabord par celle de la Parole; qui, comme objet de mon travail, doit marcher la premiere, me conduire par la liaison des parties \& la suite des conséquences jusqu'au dernier période de l'ouvrage.

17 La généralité du principe s'explique, en dernière instance, par le fait que Buffier n'entreprend pas une analyse syntaxique qui serait séparée d'une analyse sémantique. 
La PAROLE est la manifestation de la pensée par le secours des mots.

La PENSÉE nait de l'union des idées.

Les IDÉES sont les simples images des choses: mais étant intérieures \& spirituelles, il a falu, pour les faire paroitre au dehors, leur donner des corps: ce qu'on a exécuté par l'établissement des mots; auxquels on les a unies, pour qu'elles en soient l'ame \& fassent effet sur l'esprit partout où ceux-ci le font sur les sens extérieurs.

L'essence du MOT consiste à être une voix prononcée propre à faire naitre une idée dans l'esprit; \& cette propriété est ce qu'on nomme valeur; sans laquelle il ne seroit qu'un son matériel machinalement prononcé.

La VALEUR est donc, en fait de mots, l'effet qu'ils doivent produire sur l'esprit, c'est à dire la représentation des idées qu'on y a attachées: ce qui dépend de l'institution, soit commune par un usage ordinaire, soit particuliere par une suposition bien expliquée" (1747 [rééd. 1982]: 4-6).

L'axiomatisation concerne aussi des champs empiriques: Beauzée axiomatise ainsi la description des consonnes et des unités suprasegmentales ${ }^{18}$.

L'extension des matériaux empiriques à des fins de vérification peut être retracée: si Buffier (1709) dépasse Régnier-Desmarais (1705) - qui s'était cantonné au français et aux langues classiques - par l'ajout, du moins en matière de description de sons, de matériaux espagnols et italiens, Girard (1747) inclut dans sa typologie les langues slaves (et l'hébreu), et Beauzée prétend avoir consulté "des Grammaires de toute espèce: hébraïque, syriaque, chaldéenne, grecque, latine, françoise, italienne, espagnolle, basque, irlandoise, angloise, allemande, suédoise, laponne, chinoise, péruvienne" $\left(1767\right.$, p. XV), et insiste qu'il les a abordées sans a priori ${ }^{19}$. Silvestre de Sacy (1799) profite de son expérience d'arabisant, mais il a consulté aussi des grammaires du turc, du basque et du groenlandais.

Comme conclusion de cette interrogation sur l'objet de l'histoire de la grammaire/linguistique, on peut dire que celle-ci concerne la production et l'évolution de connaissances linguistiques, par des actants, qui sont en interaction dans un contexte socio-culturel donné, et qui sont aussi en rapport avec un certain passé scientifique (et culturel). Le référent historique de l'historiographie de la grammaire/linguistique est sujet à un certain nombre de variables, affectant les actants, les problèmes envisagés et traités, et le contexte: c'est à l'historiographe d'élaborer un cadre méthodologique pour cerner ces variables. L'histoire même de la discipline nous fournit les matériaux pour étoffer, dans toutes ses dimensions, ce cadre de réflexion méthodologique. Or, dans quelle mesure cette histoire nourrit-elle notre réflexion?

18 Cf. Swiggers (1984: 69-77 et 88-89)

19

Beauzée (1767: XV-XVI): "Je me suis tenu en garde contre les surprises des préjugés, contre les illusions de l'aveugle routine, contre les assertions vagues \& non éprouvées, contre les règles hasardées, contre les systèmes calqués sans modification d'une langue sur une autre: en un mot, j'ai moins compté sur les définitions $\&$ les règles des grammairiens, que sur l'analyse même des exemples qu'ils me mettoient sous les yeux". 
2. Une récurrence de faits, ou "comment l'histoire (grammaticale) peut se répéter": l'exemple de la grammaire de l'Académie

2.1. Le fait historique présente la singulière caractéristique d'être unique, tout en étant le produit de causes et de conditions générales. Sa spécificité consiste dans le "fait" que le produit ne s'explique pas par les antécédents, mais se laisse comprendre, en partie, par rapport à eux. Dans son livre passionnant Comment on écrit l'histoire, Paul Veyne a dégagé deux conclusions importantes de cet état de choses:

(1) les faits historiques forment des intrigues, non strictement chronologiques, qui sont le tissu de l'histoire: "un mélange très humain et très peu "scientifique»" de causes matérielles, de fins et de hasards;

(2) l'historien opère un découpage dans le devenir historique: sa description est partielle, elle s'attache au spécifique, et veut être un récit véridique.

Adhérant à une conception de la science comme activité intellectuelle procédant par explication en termes de lois, Veyne se voit obligé d'affirmer que l'histoire n'est qu'une compréhension du vécu ("telle est l'explication historique: toute sublunaire et pas scientifique du tout: nous lui réserverons le nom de compréhension"; Veyne 1978: 68): il n'y a pas de lois de l'histoire, il y a seulement des lois en histoire. Or, ces lois - économiques, sociales, physiques — n'expliquent pas, dans leur sommation, le fait historique: le "sublunaire" - le monde vécu - ne cö̈ncide pas avec les découpages des objets abstraits de la science (Veyne 1978: 157). En même temps, l'histoire a "peu à attendre de la science", qui est "très pauvre et se répète terriblement" (Veyne 1978: 171):

"Comme la philologie, ou encore comme la géographie, l'histoire est une "science pour nous", qui ne connaît la véritable science que dans la mesure où celle-ci intervient dans le vécu. Elle ne met d'ailleurs aucune complaisance esthétique ou anthropocentrique à s'en tenir à ce point de vue; si, pratiquement, elle pouvait échanger la doxa contre l'épistémé, elle n'hésiterait pas à faire l'échange (...) L'histoire est un palais dont nous ne découvrons pas toute l'étendue (nous ne savons pas combien il nous reste de non-événementiel à historiciser) et dont nous ne pouvons voir toutes les enfilades à la fois; aussi ne nous ennuyonsnous jamais dans ce palais où nous sommes enfermés. Un esprit absolu s'y ennuierait, qui en connaîtrait le géométral et n'aurait plus rien à découvrir ou à décrire. Ce palais est pour nous un véritable labyrinthe; la science nous donne des formules bien construites qui nous permettent d'y trouver des issues, mais qui ne nous livrent pas le plan des lieux" (Veyne 1978: 179).

L'histoire humaine, elle, ne se répète pas; mais il y a, nous semble-t-il, des récurrences - dans les actions, dans les intentions, dans la construction d'intrigues. On se demande d'ailleurs si le clivage auquel Veyne adhère - celui entre l'histoire et les "sciences" - tient debout pour l'histoire des disciplines: l'historiographie serait-elle aussi soumise à l'opposition entre "sciences" humaines et sciences?

La part d'humain que véhicule toute science transparaît, dans des degrés différents et dans des tonalités divergentes, à travers les produits de la communaute "scientifique": on la retrouve dans les hésitations théoriques, dans la mise en place de circuits de communication, dans la hantise de l'originalité, dans les discussions polémiques. Il 
s'agit là de récurrences - et il y en d'autres, comme par ex. le lancement de revues, la création de sociétés - qui rendent complexe le partage entre l'unique et le commun, entre le singulier et le pluriel.

Dans cette deuxième partie, on étudiera un exemple de récurrence différentielle dans l'histoire de la grammaire française: la récurrence réside dans le fait qu'un produit grammatical de l'Académie française se trouve être jugé, face au grand public, par un "professionnel". L'aspect différentiel réside dans la diversité des produits, dans le ton différent des comptes rendus, enfin dans l'enjeu même de la discussion critique.

2.2. L'article 26 des statuts de l'Académie française formulait comme tâches: "Il sera composé un Dictionnaire, une Grammaire, une Rhétorique, et une Poétique sur les observations de l'Académie". Si les académiciens réussissent à sortir leur Dictionnaire avant la fin du $17^{\mathrm{e}}$ siècle (1694), ils ne pècheront pas par excès de zèle dans la rédaction d'une grammaire. La Grammaire "officielle" de l'Académie se fera attendre jusqu'en 1932, mais il faut reconnaître qu'en 1705 une grammaire bâtarde était sortie de la Coupole. C'est le secrétaire perpétuel, Régnier-Desmarais, qui en assuma la responsabilité. Ayant soumis diverses portions de sa grammaire en préparation à ses collègues, l'abbé "Pertinax" s'opposa à certaines critiques et refusa d'adopter le plan que l'Académie voulait lui imposer. Les académiciens abandonnèrent leur secrétaire perpétuel à son entreprise et en 1705 Régnier-Desmarais publia son Traité de la grammaire françoise, pourvu d'une dédicace aux Quarante ${ }^{20}$. En 1706 une deuxième édition, légèrement remaniée, vit le jour. C'est la deuxième édition (1706) de la grammaire de Régnier-Desmarais qui a fait l'objet d'un long compte rendu par le Père Buffier dans les Mémoires de Trévoux (1706, pp. 1641-1671) ${ }^{21}$. Ce compte rendu a été publié en 1706, trois ans avant la parution de la Grammaire françoise sur un plan nouveau de Buffier, travail très original et qui témoigne d'une familiarité peu commune avec diverses langues européennes.

Le compte rendu de Buffier est très objectif: la recension présente la structure de l'ouvrage commenté, fait connaître les vues de Régnier-Desmarais avant d'y ajouter des remarques, et situe la grammaire dans son contexte historique. Buffier commence d'ailleurs par mettre en relief la caution dont la grammaire peut se prévaloir:

"C'est par l'ordre de l'Academie Françoise, comme nous l'apprennent les premiers mots de l'Epître dédicatoire, que ce gros Volume a été fait. Elle n'a pû y travailler en corps à ce qu'on ajoûte dans la Préface, plusieurs Architectes ne pouvant travailler, dit-on, sur le plan d'un même Edifice. Mr. l'Abbé Regnier a employé à celui-ci cinquante ans de Reflexions sur nôtre langue, la connoissance des langues voisines, \& trente quatre ans d'assiduité dans les Assemblées de l'Academie, où il a presque toûjours tenu la plume" (pp. 1641-1642).

20 Cf. Swiggers (1985).

21 Tous les renvois sont au texte original. 
Buffier discute ensuite quelques "réflexions" de Régnier-Desmarais, qui concernent la phonétique et la morphologie du français (la grammaire ne comportant pas de partie syntaxique). Les critiques de Buffier concernent en premier lieu l'attachement trop strict à la langue écrite: il convient de distinguer nettement figure (ou caractère) et son.

“(...) la prononciation de nôtre $e$ ouvert s'écrit également par $\hat{e}$, comme dans fête, par és comme dans procés, par ais comme dans niais, par est, comme dans forest, par ets comme dans balets, par oist comme dans paroist, par oye comme dans monnoye, par ayent comme ils ayent, par oient comme auroient, \&c." (p. 1646).

Un deuxième point de critique concerne le modèle latinisant qu'adopte RégnierDesmarais:

"Les Articles sont des particules declinables, qui précedant toûjours le nom servent à en faire connoître le genre \& le nombre. On observe encore ici que dans le François les cas des Noms ne different que par les Articles, mais le genitif $\&$ l'ablatif ayant en nôtre langue le même Article, pourquoi les distinguer? C'est, dit-on, que cela doit être, \& par rapport au sens, \& par rapport à la construction: par rapport au sens, l'ablatif sert à marquer séparation, privation, ce que ne fait pas le genitif: par rapport à la construction, le genitif $n$ 'est jamais regi que par un Nom; au lieu que l'ablatifl'est presque toûjours ou par quelque Nom ou par quelque Verbe. Avec toutes ces bonnes raisons, je ne sçai s'il ne seroit point plus commode pour ceux à qui l'on veut enseigner nôtre Langue de ne leur point distinguer le genitif de l'ablatif; puisque ce seroit leur abreger le travail, \& qu'en fait de langage c'est l'expression même qu'on cherche bien plus que la raison de l'expression" (pp. 1653-1654).

Homme pratique, Buffier est sceptique à l'égard des définitions trop compliquées de certaines parties du discours ${ }^{22}$, et il n'admet pas que la grammaire soit séparée de la langue (ou de l'usage langagier):

"On se sert de que pour à qui \& de qui, c'est à vous que je parle, c'est de vous qu'on parle. Dans une infinité de phrases semblables, dit-on ici, l'usage de la langue est au-dessus des regles de la Grammaire: mais la Grammaire \& ses regles sont-elles autre chose que des observations sur ce qui est en usage dans les langues, \& ne peut-on pas dire au regard des phrases précedentes qu'il n'y a rien contre les regles les plus exactes de la Grammaire, puisqu'elle observe seulement que dans le François que est aussi bien le genitif \& le Datif du Pronom qui, que de qui ? On pourroit ajoûter à ces observations l'inclination extraordinaire de nôtre langue pour cette syllabe que" (p. 1662).

Enfin, Buffier critique l'évaluation normative par Régnier-Desmarais de certains faits d'usage et signale des divergences dans les jugements d'acceptabilité:

22 “En effet à qui ne sçaura pas d'ailleurs ce que c'est que Pronom, le lui fera t'on entendre bien facilement en luy disant, que c'est une partie d'Oraison qui reçoit difference de genre, de nombre \& de cas, comme le Nom \& qui sert quelquefois à marquer par lui même une personne ou une chose, mais dont l'usage le plus ordinaire est de servir à la place du Nom d'une personne ou d'une chose, \& qui alors a toûjours la même signification que le Nom au lieu duquel on l'employe? Des esprits moins penetrans aimeroient peut-être mieux l'ancienne définition bien que moins exacte, mais plus courte" (pp. 1659-1660). 
"M. l'Abbé Regnier après ces préceptes revient encor au futur du Verbe coudre qu'il a dit être je coudrai, ajoûtant que je couserai est tellement dans la bouche de la plûpart des femmes, qu'elles se servent rarement de l'autre; mais que c'est un abus: bien des femmes ne prétendront-elles pas à leur tour traiter d'abus une expression qu'on voudroit préferer, à celle qui est la plus en usage parmi elles, à qui il appartient plus qu'aux hommes de sçavoir ce que c'est que coudre? (...)

Si quelques-uns ne sont pas toûjours du sentiment de l'Auteur, c'est qu'en fait de langue \& sur tout de langue vivante, on n'a jamais veu tout le monde d'accord. Ainsi il ne faudroit pas s'étonner que plusieurs ne disent pas avec lui mol \& effeminé, au lieu de mou \& effeminé, bisson, au lieu de buisson; lui seyent bien, au lieu de lui sieyent bien; le Bocace \& le Petrarque, au lieu de Bocace \& Petrarque; \& un grand nombre d'autres expressions semblables qu'il n'est pas necessaire de rapporter" (pp. 1668-1669, 1670-1671).

Discussion courtoise, commentaires élogieux, absence de polémique: autant de caractéristiques qui font du compte rendu de Buffier un métatexte de présentation, d'accompagnement pour un public général. Il n'en ira pas de même deux siècles plus tard quand l'Académie française, assumant cette fois-ci sa responsabilité entière, se décide à publier sa Grammaire.

2.3. En 1932 paraît la Grammaire de l'Académie française. L'ouvrage est fort médiocre, mais les professionnels auraient pu garder le silence. Ferdinand Brunot, professeur en Sorbonne, agrégé de grammaire, et connu par sa monumentale Histoire de la langue française et par ses attaques contre la grammaire scolaire, symbolisées dans son ouvrage théorique La pensée et la langue $(1922)^{23}$, ne peut se taire et publie, la même année, un ouvrage-pamphlet, qu'il importe d'analyser comme document historique et comme travail grammatical.

Dans ses Observations sur la Grammaire de l'Académie française, Ferdinand Brunot (1932) élabore une critique, qui à en juger par la présentation matérielle de l'ouvrage est parfaitement homogène en elle-même, et qui se fait en bordure exacte du texte. En effet, l'ouvrage consiste essentiellement - si l'on fait exception de la préface et de la conclusion - d'une juxtaposition, sur deux colonnes, de passages extraits de la Grammaire de 1'Académie et des critiques de Brunot visant les passages en question ${ }^{24}$. Mais le débat n'est pas en parallélisme parfait; s'il est vrai que Brunot exerce parfois sa critique sur des points précis de la grammaire des Académiciens, et s'il se moque de la simplicité, voire de la naïveté de certaines définitions ${ }^{25}$, la critique de Brunot a

23. Sur les conceptions de Brunot, voir Chevalier (1991) et Melis - Swiggers (1992).

24 Dans la suite nous utilisons l'abréviation GA [pour la Grammaire de l'Académie française] et B [pour Brunot 1932], chaque fois quand les deux textes sont confrontés (les abréviations sont suivies de l'indication des pages). Ailleurs, nous renvoyons au texte de Brunot (par ex. à la préface ou à la conclusion) comme "Brunot (1932)".

$25 \mathrm{Cf}$. [GA, 1]: "Le rôle des grammairiens se borne à dégager ces règles de l'observation du langage vivant"; $[\mathrm{B}, 10]$ : “Quel est ce langage vivant? Celui qui se parle ou celui qui s'écrit? Le premier a été à peu près complètement négligé dans cet ouvrage. Si les grammairiens doivent "dégager les règles de l'observation du langage vivant", comment expliquer la présence dans cette Grammaire de toutes les règles concernant l'emploi du subjonctif imparfait, alors que cet imparfait, sauf dans les verbes avoir et être, et à la troisième personne 
une portée plus ample: il y va de montrer que la grammaire normative en France est trop souvent une occupation de gens bornés ou arriérés, et que la science du langage en France est dans un état déplorable ${ }^{26}$. Les "vieilles erreurs" que Brunot dénonce dans sa préface ne sont pas nommées explicitement, mais à en juger par l'énergie avec laquelle Brunot s'en prend à la "méthode" des sous-entendus, on ne peut éviter la conclusion que Brunot veut en finir avec l'ancienne alliance de la grammaire et d'une logique sémanticiste très floue.

Citons à ce propos un exemple, parmi tant d'autres ${ }^{27}$. L'Académie avait formulé l'observation que parfois la proposition principale peut être entièrement sous-entendue, "quand ce qu'elle devrait énoncer résulte si évidemment de la proposition subordonnée que l'exprimer en toutes lettres devient inutile: Hélas! si j'avais pu savoir! Dire que nous nous sommes donné tant de mal! Moi qui croyais avoir fini!" [GA, 212]. Le commentaire de Brunot ne manque pas de sarcasme:

"Ici je me sens vraiment humilié; je ne découvre pas du tout ce que la principale devrait énoncer, et qui résulte si "évidemment" de la subordonnée. Pour le premier exemple: Hélas, si j'avais pu savoir, j'hésite entre: $j$ 'aurais mis ma cravate neuve, ou: je me serais suicidé. Mais pour la seconde, je ne trouve absolument rien. Pour la troisième, pensant à l'examen que je fais de ce livre de l'Académie, je suis tenté d'ajouter: et j'ai encore vingt-six pages à voir et peut-être cinquante erreurs à relever! Or il y a gros à parier que ce n'est pas à ce sous-entendu qu'a pensé l'Académie. En outre, ce n'est pas là une proposition principale, dont dépendrait la subordonnée" [B, 99-100].

C'est dans sa conclusion, où il tient un plaidoyer pour une collaboration entre écrivains et théoriciens de la grammaire - ceux-là mettant à profit leur savoir linguistique en tant qu'usagers "en contact avec la réalité vivante", ceux-ci fournissant "les cadres, la théorie, les principes généraux" - , que Brunot vomit sa bile contre la logique (ou l'espèce de logique) qu'il avait déjà décriée dans La pensée et la langue (Brunot 1922):

“À l'histoire et à la psychologie, qui expliquent tout ou à peu près, on substitue la logique, qui n'explique presque jamais rien. De là l'emploi perpétuel de cette méthode des sousentendus, qui permet de ramener toute proposition, à coups d'ellipses, à la proposition, théorique et complète, telle que les grammairiens de la Grammaire générale et philosophique l'avaient établie. On demande “Qu'avez-vous?" Si je réponds: Rien, c'est que j'ai dans l'esprit: Je n'ai rien. Si j'ajoute: Je voudrais sortir, c'est que je pense intérieurement: si je pouvais. Une mécanique formelle, décorée du nom d'analyse, empêche de suivre la démarche réelle de la pensée, ankylosée dans une attitude hiératique. On complète, on redresse; c'est toute une orthopédie. La doctrine des restrictions mentales, appliquée au langage, devient la doctrine des additions mentales" (Brunot 1932: 126).

des autres verbes, est à peu près sorti de l'usage?", ou [GA, 3]: "Le mot est un signe qui représente une image, une idée ou un mode quelconque de l'esprit"; [B, 11]: "Que signifie "un mode quelconque de l'esprit"? Estce la folie? la raison? l'exaltation? la réflexion?"

26 "Il fallait aussi montrer aux malveillants - il y en a - que la science du langage n'en est pas en France au point où on pourrait la croire, si on en jugeait d'après une cuvre à laquelle aucun homme de métier n'a mis sa marque ni donné son nom" (Brunot 1932: 7-8).

27 Cf. aussi les observations de Brunot (1932: 56, 62 [à propos du comparatif], 85, 115-116). 
Il semble donc que Brunot, exigeant de la grammaire qu'elle soit explicative, considère l'histoire et la psychologie comme des bases sûres. Il est vrai que la Grammaire de l'Académie ne témoigne guère de connaissances approfondies en grammaire historique chez les Immortels, et Brunot n'omet pas de déplorer l'absence de ce supplément d'âme (grammaticale) ${ }^{28}$. Mais il convient de se demander si c'est vraiment là qu'achoppe la Grammaire de l'Académie. En effet, ne pas faire la distinction entre ce qui est emprunt et ce qui est héritage (continu) peut choquer l'esprit de l'historien de la langue (cf. GA, 9 et B, 17-18; GA, 201-202 et B, 93) ${ }^{29}$, mais il faut reconnaître que la description grammaticale n'en est pas hypothéquée. Le manque de perspective diachronique affecte plus sérieusement la conception de la dérivation, peu exacte, chez les Académiciens, mais là aussi les dégâts sont limités:

[GA, 13-14]: [I] "Un grand nombre de mots composés, [sont formés par la réunion] ... de racines latines ou grecques juxtaposées: homicide, fébrifuge, mammifêre, biographe, bibliophile, hectogramme (...) [II] Un mot peut être à la fois composé et dérivé: dans sou-terr-ain, extra-vag-ance, im-poli-ment, on reconnait un préfixe, un radical et un suffixe";

[B, 21 ]: [ad I] "Homicide est un mot latin, emprunté celui-là, et non un composé français. Phile qui entre dans bibliophile et gramme, qui existe indépendamment d'hectogramme sont, non pas des racines, mais des mots grecs. [ad II] Sans doute, mais les faits sont em-brouillés ici comme à plaisir; extravag-ance a été tiré d'extravag-ant et d'extravagu-er, comme impoliment d'impoli, tandis que souterrain a été formé par addition simultanée à terre du préfixe sous et du suffixe ain, à l'imitation du latin subterraneus. Il n'y avait pas de mot souterre" 30 .

Enfin, à d'autres endroits une meilleure connaissance de la diachronie aurait permis une plus fine description de l'état actuel de la langue: déclarer la forme pis un adverbe, cela est exact, mais Brunot - rappelant son statut d'ancien neutre - est capable d'expliquer les structures cela est encore pis et quelque chose de pis, rien de pis, quoi de pis. On pourrait toutefois rétorquer que Brunot aurait pu envisager la question de façon plus englobante, en traitant à la fois de mieux et de bien.

28 “J'ajoute qu'il serait bon qu'aux connaissances théoriques les auteurs de la grammaire future joignissent au moins quelques notions d'histoire. Pour faire un exposé, même statique, d'une langue à une époque donnée, il est indispensable de savoir d'où elle vient. Sinon, le sens de la vie étant absent, on risque de se méprendre totalement sur les faits, leur caractère et leur valeur" (Brunot 1932: 125).

29 Ou provoquer des réactions ironiques, voire sarcastiques: [GA, 10]: "Il y a toujours entre les doublets une parenté de sens que l'étymologie permet de retrouver"; [B, 18]: "Cette parenté éclate dans la phrase: avoir passé sa vie dans un hôtel et finir dans un hôpital! Il est fâcheux que les pensionnaires de l'Assistance Publique n'aient pas conscience de ce rapport"; [GA, 65]: "Mais il est incorrect de dire: Ce n'est pas rien, pour dire: C'est quelque chose, cette formule signifiant littéralement: Ce n'est pas quelque chose, ce n'est rien, soit le contraire de ce qu'on veut dire"; [B, 52]: "Tout le raisonnement, fondé sur le sens primitif de rien, repose sur une erreur. Rien a ici son sens ordinaire et moderne de nulle chose, néant. Je dirai donc fort bien: Cette grammaire n'est pas rien, elle est quelque chose, mais quelque chose de très imparfait'. À d'autres endroits, les rares incursions dans l'histoire de la langue révèlent l'ignorance crasse des Académiciens $(B, 85$ à propos des participes; B, 88 à propos des adverbes confusément, énormément).

30 On appréciera la pertinence de la remarque de Brunot pour une théorie (cyclique ou à paliers) de la dérivation, comme elle a été développée dans le cadre générativiste ou dans celui de la phonologie lexicale. 
Car ce que Brunot reproche à la Grammaire de l'Académie, c'est qu'elle n'a pas de doctrine. À propos du problème de l'accord de supposé, attendu, ci-joint, étant donné, il observe:

"C'est ici qu'on voit combien il eût été utile de ne pas émietter des observations empiriques, et de considérer les faits d'un peu haut, de façon à avoir une doctrine" (Brunot 1932: 86).

Si Brunot n'explicite guère la notion de "doctrine", il nous semble toutefois possible d'inférer quelques-unes de ses caractéristiques (stipulatives), à partir d'une lecture du texte:

(a) une doctrine grammaticale doit être cohérente;

(b) une doctrine grammaticale doit s'appuyer sur une solide connaissance de l'histoire de la langue et ne doit négliger aucun fait observé dans la langue vivante;

(c) une doctrine grammaticale doit se concrétiser dans un plan de description et dans des règles précises.

C'est par rapport à ces exigences que s'exerce la critique de Brunot.

(a') La description grammaticale des Académiciens souffre d'incohérences: ainsi, les préfixes sont en général assignés à la classe des prépositions ou à celle des adverbes, mais de nombreux préfixes (p. ex. re-, dé-, in-) sont alors inclassables (ce qui s'explique par l'incapacité des Académiciens de fournir une analyse morphologique). De même, sous le titre "Emploi des prépositions", la Grammaire de l'Académie traite de durant pour constater qu'il conserve "sa valeur de participe et se place après le nom dans des expressions comme: Sa vie durant" (GA, 204). Brunot commente sèchement: "On reconnaît qu'ici durant est un participe; alors pourquoi l'appeler préposition et disserter sur sa place? On dit sa vie durant pour signifier aussi longtemps que sa vie durera. Ceci n'a rien à voir avec la place qu'il convient de donner aux prépositions" $(\mathrm{B}, 95)$.

(b') La Grammaire de l'Académie française ne pèche pas seulement par cécité diachronique; elle passe à côté de certains faits réels. Ainsi, les temps surcomposés sont exclus du tableau des formes verbales ${ }^{31}$ et à propos de la vue cavalière que la Grammaire donne des verbes défectifs, Brunot note: "Ces omissions ont le grave tort de masquer complètement la morphologie réelle de la langue moderne" (Brunot 1932: 76). À d'autres endroits, la Grammaire informe mal sur la langue actuelle ${ }^{32}$ :

[B, 64]: “Je ne connais pas l'expression la reine feue, la République feue. Ce sont des créations qui ne peuvent manquer de réussir" [à propos de GA, 93];

31 Cf. Brunot (1932: 80-81).

32 Cf. aussi Brunot (1932: 73; correction de ressortir de en ressortir à) et Brunot (1932: 107-108; emploi des subjonctifs imparfaits): "Or il y a des pages et des pages de ces schibboleths: que nous rissions, que vous luisissiez, que nous naquissions, que nous convainquissions. Ceux qui aiment l'harmonie pourront ajouter: que vous vous décarcassassiez. Confississions, qu'on lisait dans le premier tirage (p. 146) était une faute d'impression. Un papillon en corrigeait une semblable: suffississions (p. 156). Mais le fait même qu'elles se sont glissées dans les tableaux de conjugaison prouve que l'Académie s'est perdue dans les marécages en suivant des feux-follets. On donne beau jeu aux gouailleurs!" (Brunot 1932: 108). 
[GA, 193]: “On ne dit pas, ou on ne devrait pas dire: J'ai très faim, J'ai très soif, C'est très dommage"; [B, 89]: "Et l'Académie prétend légiférer au nom de l'usage!"

(c') Quant au plan, la Grammaire de l'Académie se caractérise par une disproportion entre la morphologie (avec 59 pages de conjugaisons) et la syntaxe (29 pages au total). De plus, le plan adopté n'est guère justifié ou explicité. Les règles, en général peu profondes, sont à certains endroits tout à fait insuffisantes, et Brunot épingle patiemment les dérapages:

- l'élision de ce devant le verbe avoir n'est pas relevée (Brunot 1932: 16);

- la liaison ne fait pas toujours "entendre la consonne finale" du premier mot (cf. un grand homme - ici se pose tout un problème de description morphophonologique) ${ }^{33}$;

- [GA, 18]: "Dans certains noms, cette forme [du masculin] subit une modification devant l'e du féminin: épicier, épicière; musicien, musicienne; ... héros, héroïne; neveu, nièce"; [B, 24]: "Cette 'modification' devant $e$, est une des choses les plus admirables parmi toutes celles que nous enseigne cette Grammaire. Devant $e$ muet, c'est-à-dire sans valeur phonique, héros devient héroüne et neveu, nièce. Il est regrettable que ce miracle de l'e muet ne soit pas expliqué";

- l'emploi de celui/celle devant un adjectif ou un participe est condamné à tort comme une incorrection ${ }^{34}$;

- [GA, 92]: "Certains adjectifs comme $n u$, mi ... plein, franc restent invariables quand ils précèdent le nom et s'accordent avec le nom quand ils le suivent"; [B, 63]: "Ainsi formulée, la règle est absurde. On ne dit pas en plein séance, ni une franc gaieté, ni la haut mer";

- les adverbes ne peuvent modifier, selon l'Académie, qu'un verbe ou un participe (GA, 190);

- selon la Grammaire de l'Académie, le temps du verbe de la proposition principale déterminerait le temps du verbe de la subordonnée; Brunot corrige: "C'est ici une des règles les plus chères à l'Académie et il faut bien le dire, une des plus étroites et des plus fausses. Il est bien vrai que, suivant des concordances de pensée, des rapports s'établissent, mais les formes ne se correspondent point nécessairement" (Brunot 1932: 104-105).

Observation peu rigoureuse des faits de langue, conception bornée du travail grammaticographique, manque de systématisation ${ }^{35}$ : à ces défauts s'ajoute une sensibilité

33 Cf. Brunot (1932: 16).

34 Brunot (1932: 46); cf. aussi p. 49 à propos de quoi.

35 Un bel exemple en est fourni par Brunot (1932: 117-120), réécrivant le bref paragraphe sur l'interjection [GA, 200] en une analyse sémantique et syntaxique des types d'interjection.

36 Cf. Brunot (1932: 36): “C'est un des chapitres où se montre le plus nettement le parti-pris de rester à la surface, je veux dire d'examiner les constructions sans faire aucune allusion au sens et à la valeur qu'elles ont. Et cela fausse tout". 
peu aiguisée pour les "finesses" de la langue. Brunot, attentif au sens et à la valeur des constructions $^{36}$, critique la Grammaire - et rejoint par là les intuitions de Gustave Guillaume - , pour son analyse trop sommaire et peu éclairante de la détermination:

- [GA, 29]: "L'article est souvent omis devant le nom d'attribut: Tu seras soldat"; [B, 34]: "On eût aimé quelques éclaircissements sur la différence de sens entre tu seras soldat et tu seras un soldat. Napoléon était homme, comme nous tous; Napoléon était un homme";

- [GA, 38]: “On peut supprimer l'article devant certains noms de pays précédés d'une préposition ou employés dans un sens général"; [B, 42]: “Toujours le 'sens général'! On demanderait pour que cette règle fût compréhensible, qu'il fût établi une distinction précise entre les villes d'Allemagne et les villes de l'Allemagne" 37.

De même, la Grammaire de l'Académie ne rend pas compte des différences de distribution générale ou universelle (globalisante vs discrétisante) qui opposent tout et chaque: "tout ... s'emploie au singulier, sans article, dans le sens de chaque" (GA, 80; critique $B, 59$ ).

Jusqu'ici nous avons anailysé le texte de Brunot "en amont", dans son repli critique sur la Grammaire de l'Académie; il faut reconnaître que le texte de Brunot présente aussi une dimension "en aval" par les perspectives qu'il ouvre - sans les théoriser pour une "autre" conception, plus scientifique, de la grammaire. On sait que dans $L a$ pensée et la langue, Brunot avait développé une telle conception, sans parvenir à en distiller une grammaire. Ce qu'il nous livre dans ses Observations sur la Grammaire de l'Académie française sont des bribes d'un système grammatical, dont il est peutêtre utile de dégager certains aspects théoriques, non explicités par Brunot.

Ces bribes de systématisation sont formulées, rappelons-le, dans une forme "inversée": Brunot n'énonce pas des principes théoriques, il les insère subrepticement dans son discours critique. Ces principes, il faut les dégager par une lecture "entre les lignes" du texte, et il faut leur attribuer une place dans l'économie d'un système théorique qui n'est pas fourni. Lecture interprétative qui risque d'être incomplète et qui risque de forcer le texte de Brunot, mais on ne peut nier la présence de certains "indices".

Ainsi, Brunot a vu clair dans au moins trois problèmes fondamentaux de la description grammaticale:

(1) la fonction des catégories qu'on établit;

(2) le statut de certains éléments et leur répartition à l'intérieur des catégories;

(3) la prise en compte de "processus intercatégoriels".

La fonction des catégories est un problème que Brunot relève à propos du pronom: souvent celui-ci n'est pas un "lieu-tenant" du nom, mais un "nominant", qui sert en même temps de support à la forme conjuguée du verbe. 
"Cependant on ne se décide pas à aller jusqu'au bout et à déclarer franchement que dans: je parle, je est simplement une forme de conjugaison destinée à marquer la première personne. Il est donc faux de dire que le plus souvent le pronom tient la place du nom. Je, tu, n'ont jamais ce rôle" (Brunot 1932: 43);

"Aucun de ces mots n'est proprement pronom, puisqu'aucun ne représente un nom: On dit; quelqu'un vous demande; nous parlions de quelque chose; les biens d'autrui" (Brunot 1932: 49-50).

Toute grammaire qui établit des classes de mots doit affronter le problème du statut de certains éléments, par rapport à leur assignation à une classe ou par rapport à leur répartition à l'intérieur d'une classe. Brunot a vu que les numéraux ne constituent pas une classe unique ${ }^{38}$, et que la réanalyse, en classes de mots, des locutions prépositives ou conjonctives mène à des incohérences ${ }^{39}$. Mais c'est surtout la répartition des éléments qui retient son attention; ainsi, à propos des pronoms compléments, il propose une nouvelle classification.

"Il eût fallu classer et distinguer: $1^{\circ}$ les formes qui ne peuvent pas suivre le verbe, $m e, t e ; 2^{\circ}$ celles qui se mettent tantôt devant, tantôt derrière $(l a, l e, l u i)$, et ajouter que derrière le verbe, mais là seulement, elles portent l'accent tonique: regarde-le, essaie-la, parle-lui"'(Brunot 1932: 44-45).

Quant aux processus intercatégoriels, Brunot n'en parle pas explicitement, mais il en a repéré au moins trois, pas toujours identifiés par les grammairiens:

(1) celui, peu grammaticalisé (moins en tout cas qu'en ancien français), de la concomitance:

[GA, 185]: "Le verbe se met au pluriel quand il a plusieurs sujets ... réunis par une conjonction ou par une préposition";

[B, 87]: "Un exemple n'eût pas été de trop. On a pensé sans doute à des phrases comme: Le singe avec le léopard, gagnaient de l'argent à la foire".

(2) celui de la synapse de catégories, reconnaissable à une insertion syntagmatique peu uniforme et à une morphologie déviante:

[GA, 25]: "Les noms composés qui s'écrivent en un seul mot prennent généralement la marque du pluriel à la fin du nom ... sauf monseigneur, madame, mademoiselle, qui font messeigneurs, mesdames, mesdemoiselles";

[B, 32]: "Cela est exact, quand monseigneur et les autres sont employés comme titres, mais non pas quand ils servent de noms. On ne dit pas des mesdames, des mesdemoiselles, mais simplement des dames, des demoiselles. Que si par ironie on les emploie au pluriel, on dit des madames, des mademoiselles".

(3) enfin celui, mieux connu, de la transposition intercatégorielle, comme celle du nom propre au nom commun ${ }^{40}$ ou celle du nom commun au pronom:

38 Cf. Brunot (1932: 53-54).

39 Cf. Brunot (1932: 93-94).

40 Cf. Brunot (1932: 29). 
[GA, 20]: "Personne, chose, féminins quand on les emploie pour désigner une personne ou une chose déterminée, deviennent masculins quand on les prend au sens indéterminé: personne n'est parfait, quelque chose de nouveau";

[B, 25]: "Exact, mais alors ce ne sont plus des noms. N'eût-il pas mieux valu dire: quand ils cessent d'être des noms".

2.4. Concluons. L'étude d'un exemple historique, individuel permet-elle de dire quelque chose au-delà du fait même? Au moins ceci: un fait historique a des analoga, ou, en d'autres termes, certaines intrigues se ressemblent: par leurs acteurs, par les stratégies mises à l'emploi, par le contexte d'insertion, par la chaîne même des événements.

Nous avons étudié comme exemple la réception, très critique, de la Grammaire de l'Académie française par Ferdinand Brunot, l'un des grammairiens français le plus en vue à l'époque: à la caution d'une institution s'oppose le bon sens et la compétence grammaticale (et grammaticographique) d'un théoricien de la langue. L'intrigue avait un précédent: le compte rendu de la grammaire de Régnier-Desmarais, secrétaire perpétuel de l'Académie, par le P. Buffier. Mais la qualité des produits et sans doute aussi le tempérament des critiques ont fait que les "résultats" des intrigues diffèrent sensiblement. Pourtant on retrouve, à plus de deux siècles de distance, des exigences de méthode semblables: clarté des définitions et sûreté de l'observation.

\section{Références bibliographiques}

ALBRECHT, J. éd. 1975. Pierre-Nicolas Bonamy: Vier Abhandlungen zum Vulgärlatein und zur Frühgeschichte des Französischen. Tübingen.

BEAUZÉE, N. 1767. Grammaire générale, ou exposition raisonnée des éléments nécessaires du langage, Pour servir de fondement à l'étude de toutes les langues. Paris.

BREKLE, H.E. 1985. Einführung in die Geschichte der Sprachwissenschaft. Darmstadt.

BRUNOT, F. 1922. La pensée et la langue. Méthode, principes et plan d'une théorie nouvelle du langage appliquée au français. Paris.

-. 1932. Observations sur la Grammaire de l'Académie française. Paris.

BUfFIER, Cl. 1706. compte rendu de Régnier-Desmarais (deuxième édition, 1706). Mémoires de Trévoux année 1706. 1641-1671.

-. 1709. Grammaire françoise sur un plan nouveau. Paris.

Bunge, M. 1974. Treatise on Basic Philosophy, vol. 1: Semantics: Sense and Reference; vol. 2: Semantics: Interpretation and Truth. Boston - Dordrecht.

Chevalier, J.-C. 1991. "Ferdinand Brunot (1860-1937), La Pensée et la Langue". Dans: H. Huot (éd.), La grammaire française entre comparatisme et structuralisme 1870-1960, 73-114. Paris.

COHEN, M. 1958. La grande invention de l'écriture et son évolution. Paris.

DIRINGER, D. 1948. The Alphabet: A Key to the History of Mankind. New York.

-. 1962. Writing. London.

FÉVRIER, J. G. 1948. Histoire de l'écriture. Paris.

Foucault, M. 1966. Les Mots et les Choses. Paris. -. 1969. L'archéologie du savoir. Paris.

GELB, I.J. 1969. A Study of Writing. Chicago.

GIRARD, G. 1747. Les Vrais Principes de la langue françoise: ou la parole réduite en méthode, conformément aux loix de l'usage. Paris. [Réédition, avec une introduction par P. SWIGGERs. Genève, 1982]

Granger, G.-G. 1960. Pensée formelle et sciences de l'homme. Paris.

-. 1968. Essai de philosophie du style. Paris. 
Greimas, A. J. 1976. “Sur l'histoire événementielle et l'histoire fondamentale”. Dans: A. J. GrEImAS, Sémiotique et sciences sociales, pp. 161-174. Paris.

HARRIS, Z.S. 1965. String Analysis of Sentence Structure. The Hague.

Melis, L. - Swiggers, P. 1992. "Ferdinand Brunot contre la sclérose de la grammaire scolaire". Cahiers Ferdinand de Saussure 46. 143-158

Mounin, G. 1967. Histoire de la linguistique des origines au XXe siècle. Paris.

PEDERSEN, H. 1916. Et blik paa sprogvidenskabens historie, med saerlight hensyn til det historiske studium av sprogets lyd. Copenhague. [Trad. anglaise: A Glance at the History of Linguistics. With particular regard to the history of phonology. Amsterdam, 1983]

-. 1924. Sprogvidenskaben $i$ det nittende aarhundrede. Metoder og resultater. Copenhague. [Trad. anglaise: Linguistic Science in the Nineteenth Century. Cambridge, 1932; réédition en 1962 sous le titre The Discovery of Language. Bloomington]

RÉGNIER-DESMARAIS, F.-S. 1705. Traité de la grammaire françoise. Paris. [Deuxième édition, 1706]

RoBINS, R.H. 19792. A Short History of Linguistics. London. [19671]

SCHON, D. 1963. Invention and the Evolution of Ideas. London.

SEILER, H. 1977. Sprache und Sprachen. München.

-. 1988. The Dimension of Participation. Guadalajara.

SilveSTRE DE SACY, A.-I. 1799. Principes de grammaire générale, mis à la portée des enfans, et propres à servir d'introduction à l'étude de toutes les langues. Paris.

SWIGGERS, P. 1984. Les conceptions linguistiques des Encyclopédistes. Étude sur la constitution d'une théorie de la grammaire au siècle des Lumières. Heidelberg.

-. 1985. "La grammaire française de Régnier-Desmarais". Historiographia Linguistica 12. 261-266.

-. 1991. "Creuser dans l'histoire des sciences du langage: intérêts et programmes". La Licorne 19. 115-134.

THOM, R. 1974. Modèles mathématiques de la morphogenèse. Paris.

THOMSEN, V. 1902. Sprogvidenskabens historie: En kortfattet fremstilling. Copenhague. [Trad. allemande: Geschichte der Sprachwissenschaft bis zum Ausgang des 19. Jahrhunderts. Halle, 1927].

THUROT, F. 1796. Tableau des progrès de la science grammaticale. Paris. [Rééd. par A. Joly, Bordeaux, 1970]

Toulmin, S. 1960. The Philosophy of Science. London - New York.

VEYNE, P. 1978. Comment on écrit l'histoire (suivi de: Foucault révolutionne l'histoire). Paris. [Première édition: 1971]

Povzetek

\section{SLOVNICA FRANCOSKEGA JEZIKA IN NJEGOVO ZGODOVINOPISJE: KRITIČNI ODZIV V ČASOVNEM LOKU DVEH STOLETJIH}

Prispevek je razmišljanje o spoznavanju in veljavnosti zgodovinopisja francoske slovnice; temu pa sledi izkustvena študija.

V svojem prvem delu skuša natančno zamejiti predmet zgodovinopisja slovnice (in jezikoslovja): ta predmet predstavlja ustvarjanje slovničnih in jezikoslovnih znanj in to ustvarjanje se uresničuje $z$ dejavniki, ki jih vsebuje dani socialni in kulturni okvir, pogojeno pa je zmeraj tudi z znanstvenimi dognanji iz prejšnjih časov. To ustvarjanje je vezano na nekaj spremenljivk, ki lahko prizadenejo dejavnike, kontekst in splošna vprašanja, ki so obravnavana.

Drugi del prispevka ponuja analizo nekega, skoraj bi lahko rekli, ponovljenega dogajanja: kritičen odziv in pretres akademijske slovnice - po dveh stoletjih. Leta 1706 je namreč Claude Buffier predložil francoski humanistični javnosti čisto slovnično kritiko slovnice francoskega jezika, ki jo je objavila Francoska akademija, oziroma natančneje njen (dosmrtni) tajnik F. S. Régnier-Desmarais. Leta 1932 pa Ferdinand Brunot, znameniti francoski slovničar in jezikoslovec, močno kritično, mestoma celo sarkastično, ocenjuje tistega leta izišlo izdajo slovnice francoskega jezika te iste slavne ustanove. Če sta ton in način presoje obeh kritik zelo različna, pa vendar najdemo, ob časovni razliki dveh stoletij, podobne zahteve, kar zadeva metodo: jasnost v definicijah in zanesljivost pri opazovanju jezikovnih dejstev. 